\title{
Importance of Parasitic Vaccines in Integrated Control of Parasitic Diseases in Livestock
}

\author{
Hardeep Kalkal*, Sukhdeep Vohra and Snehil Gupta \\ Department of Veterinary Parasitology, College of Veterinary Sciences Lala Lajpat Rai \\ University of Veterinary and Animal Sciences, Hisar-125 004, India \\ *Corresponding author
}

\begin{tabular}{l} 
K e y w o r d s \\
$\begin{array}{l}\text { Drug resistance, } \\
\text { Integrated control } \\
\text { of parasites and } \\
\text { vaccines }\end{array}$ \\
Article Info \\
$\begin{array}{l}\text { Accepted: } \\
\text { 15 January } 2020 \\
\text { Available Online: } \\
\text { 10 February } 2020\end{array}$ \\
\hline
\end{tabular}

\section{A B S T R A C T}

Parasitic diseases have unfavorable affect on animal's health and threaten profitable animal production. These infections also play a greater role in the spread of zoonotic diseases. Parasitic infections cause severe morbidity and mortality in animals especially those affecting the gastrointestinal system and thus affect the economy of livestock owner by decreasing the ability of the farmer. Due to all these reasons proper control of parasitic infection is critically important for sustained animal production. The most common method to control parasitic infection is chemotherapy, which is very effective but has several disadvantages like drug resistance and drug residues. Integrated approaches to control parasitic infections should be prepared including grazing management, biological control, genetic resistance of hosts, and parasitic vaccines. The most sustainable and economical approach to control parasitic infection in our country is to vaccinate animals, although vaccines increase the initial cost, but the immunity offered by the vaccine are long lived. Thus, vaccination of animals for various clinical, chronic, subclinical parasitic infections will be a cheaper and effective alternative to control parasitic infection for long time and improve animal production.

\section{Introduction}

Diseases caused by parasites are responsible for most destructive and prevalent diseases in animals. Parasitic infections are related with huge economic losses in terms of production losses, losses due to infertility. Control of parasitic diseases in animals should be aimed to increase the productivity. Therefore, efficient, economic, and sustainable control methods are to be used. Despite the availability of a number of effective drugs for the treatment of most important diseases, there is need for the development of successful vaccines are not investigated. The reasons for the development of vaccines is 
due to the increasing problem of resistance of parasite, lack of newly developed effective drugs, and the presence of drug residues in milk, milk derived products.. Hence, to control parasitic diseases a multifaceted approach involving the integration of chemotherapy, grazing management, biological control, genetic resistance of hosts, and parasitic vaccines should be implemented. Many parasites have sophisticated immune evasion mechanisms making it at present difficult to conceive the development of efficient vaccines. However, the rapid development of immunology and genetic manipulation of cells will perhaps change these prospective in near future.

Economic loss due to parasitic diseases in livestock production in India

During the last 5 years incidence of gastrointestinal (GI) parasitic infections reported in India ranged between $25 \%$ and $78 \%{ }^{(1)}$. The most commonly reported GI parasitic infections include: Haemonchus contortus, Trichosrtongylus colubriformis, Paramphistomes, Fasciola gigantica, Strongyles spp., Chabertia spp., Dicrocellium spp. Nematodirus helvatianus, Bunostomum trigonocephalum, Toxocara vitulorum, Moniezia spp., Trichuris spp., Strongyloides spp., Eimeria spp., and Balantidium spp.

Economic losses due to GI parasitism in Rajasthan were Rs. 97.37 crores in adult and Rs. 21.79 crores in yearling sheep.

\section{Blueprint to control disease infections in livestock}

An integrated approach to control parasitic infections in animals consist of grazing management, breeding animals for genetic resistance against parasites, biological control, strategic chemotherapy, prophylactic vaccination $^{(2)}$.

\section{Grazing management}

Grazing management is defined as "manipulation of animal grazing practice to achieve the optimum results by reducing the parasite burden $(3,4)$." In grazing animals, main source of spread of parasitic infection is through grazing of pastures containing parasite eggs and larvae and its decontamination is very difficult. The best ways to prevent pasture infection are:

a. Controlled rotational grazing or pasture rotation system: It is a management technique involving the subdivision of pasteur, grazing of each paddock, and allows it to rest for time (5)

b. Alternate or mixed grazing: In this system two or more host species are allowed to graze together in the same field, as in any given environment two different host species do not share common parasite species. This alteration between species can be a successful tool of management.

c. Pasteur resting: Animals should not be allowed to graze in the same paddock for a longer time and preventing animals to do so is called as pasteur resting. Resting period varies from 2 months to 6 months depending upon climate. Studies conducted in semi-arid regions of Rajasthan revealed that sheep grazed during monsoon on spring contaminated, summer ungrazed pasture had very low fecal egg counts, pasture larval burden, and worm counts compared to those on continuously grazed contaminated pasture (6).

d. Zero grazing: It is an alternative system in which livestock are housed and are fed with treated forage or silage that does not allow parasite growth. This approach carry a low risk of parasitic disease requiring no control measures and is an efficient system of energy conservation and use of land resources ${ }^{(2)}$. 


\section{Breeding animals for genetic resistance against parasites}

Genetic resistance is best approach to sustainable parasite control. In Northern Australia, Bos taurus has been replaced by Bos indicus cattle as they are resistant to cattle tick and babesiosis. Nowadays research is going on the selection of breeds that are genetically resistant for GI nematode infections for both sheep and cattle ${ }^{(7,8)}$. There are over 30 indigenous breeds of cattle and 10 breeds of buffaloes in India. All these breeds show resistance to various diseases including GI parasites (2). Hence, selective cross breeding should be promoted to develop breeds that are genetically resistant to ticks and parasitic infections.

\section{Biological control}

Larsen ${ }^{(9)}$ has stated that nematophagous fungi such as Duddingtonia flagrans, Drechmeria coniospora, Harposporium anguillulae, Verticillium chlamydosporium, Arthrobotrys oligospora, Paecilomyces lilacinus naturally occur in feces of various ruminants. These fungi have the ability to infect and damage parasite eggs thus preventing the development of egg to infective larvae on pasture. As per Sanyal et al., ${ }^{(10)}$, feeding of 5,00,000 spores of the fungi $/ \mathrm{kg}$ body weight of the cattle or sheep as a bolus can significantly reduce the parasite burden. These fungi are very efficient in controlling Haemonchus spp., Trichostrongylus spp., Ascaris spp., Fasciola spp., and Ostertagia spp. of parasites.

\section{Strategic chemotherapy}

Several broad spectrum anthelmintics are currently available to control GI parasitic infection. Administering the therapeutic dose of anthelmintics during the peak season of parasitic infections can reduce the cost and maximize the benefit. Anthelmintic treatment can increase the productivity (>20\%) and result in a significant economic gain (Rs. 46 million) in milking cows ${ }^{(1)}$. Despite the great impact of anthelmintic therapy on animal production, there are several disadvantages. One of the major being drug resistance. Resistance to anthelmintic agents has been reported among all the species of GI parasites in all parts of the world including India ${ }^{(11)}$. Resistance can be prevented by the following strategies, i.e., strategic use of chemotherapy, "parasites in refugia" in grazing fields. "Parasites in refugia" means worm populations that are not exposed to anthelmintic treatment and are left to develop on pasteur ${ }^{(12)}$. Resistance can also be checked out by discovering drugs that have better and narrow spectrum of activity for parasites. Chemical residues in meat and milk are also a major disadvantage of using chemotherapy for control of parasitic diseases. To overcome these problems narrow spectrum drugs should be discovered and chemicals that are rapidly degraded into harmless metabolites should be used. This will help in reduction of drug residues in animal food ${ }^{(13)}$.

\section{Prophylactic vaccination}

Vaccines are most efficient, reliable and sustainable method, and therefore also referred as green solutions to control parasitic disease and are useful in reducing reliance on pharmacological drugs and pesticides ${ }^{(2)}$. The use of vaccines has multiple benefits such as improving animal health and welfare by controlling animal infections; improving public health by controlling zoonoses and foodborne pathogens in animals; solving problems associated with resistance to acaricides, antibiotics, and anthelmintics; keeping animals and the environment free of chemical residues and maintaining biodiversity. All of these attributes should lead to improved sustainability of animal production and increasing economic benefit 
(14). Descriptions of various parasitic vaccines are summarized below.

\section{Parasitic vaccines}

\section{Protozoan vaccines}

Protozoan infections in animals are related to significant production losses and many organisms are also responsible for zoonotic diseases or have close relationships to human parasites, thus increasing their significance as infection reservoirs for human diseases. Vaccines for human protozoa are not available yet, but a number of veterinary vaccines are being formulated and are available ${ }^{(15)}$ types of vaccines developed are:

\section{Live protozoan vaccines}

Live vaccines are based on live organisms that stimulate an immune reaction in the hosts, mimicking natural infections. In protozoan infections, the immunological mechanisms involved in protection and the stages of protozoan parasite involved in infections are mostly not have been defined therefore, most of the vaccines make use of the live organisms itself to induce the required protective immune response. A live vaccination approach induces $\mathrm{T}$-cell mediated immune responses through correct intracellular processing and presentation of antigens in association with major histocompatibility complex Class I and Class II antigens. T-cell responses are considered critical to protect against intracellular pathogens and live vaccination approaches more closely mimic the induction of both the innate and adaptive immune responses that would occur in natural infection and will therefore induce appropriate inflammatory and regulatory immune responses in the host animals (14). The haemoprotozoans display antigenic diversity in their different life cycle stages within the host as well as between different species and strains and, within the same life cycle stages due to which vaccine development for these organisms is tough ${ }^{(16)}$. These also have a high degree of genetic complexity. Depending upon the format of infection different types of live vaccines are:

a) Vaccine based on complete but shortened life cycle infection (precocious strains of Eimeria) Coccidiosis is caused by obligate intracellular protozoan parasite. These parasites undergoes a definite asexual cycles of merozoite production in the gut epithelial cells (3-4 cycles) before the production of infective stages i.e., oocysts. As the infection is self-limiting, therefore vaccination with a small dose of infective oocyst provides solid immunity against homologous infections with minimal pathology ${ }^{(15)}$.

All the vaccines present in world are based on live virulent and live attenuated formulations. The process of attenuation has been achieved by several passages in chicken embryos or by using the precocious lines i.e., oocysts selected from naturally occurring "precocious" Eimeria strains that produce less merogenic cycles and these are safer to use. The early oocyst is found to be less pathogenic because of the decreased schizont size and lesser schizogonic stages. First commercial vaccine to fight with coccidiosis in poultry was Coccivax launched in USA 1951. This vaccine contains oocysts of Eimeria acervulina, Eimeria maxima, and Eimeria mitis and was developed for broilers. Live virulent vaccines available are Coccivac B (Merk Animal Health and is prepared using E. acervullina, E. maxima, Eimeria tenella, Eimeria mivati) Coccivac D, VAC M, ADVENT and inovocox registered in the USA; Immunocox $\mathrm{C} 1$ and Immunocox $\mathrm{C} 2$ registered in Canada and Nobilis Cox ATM from Netherlands. The live attenuated vaccines include Eimeria Vac (China); Eimeria vax $4 \mathrm{~m}$ (Australia); Hipracox 
Broilers (Spain); Immuner gel coc (Argentina); Livacox Q (it is a quadrivalent live attenuated vaccine for breeders and layers against E. acervullina, E. tenella, Eimeria necatrix, E. maxima) and Livacox T (Czech Republic) ${ }^{(17)}$. Mixed vaccines consisting of precocious strain of $E$. tenella and nonattenuated strains of $E$. acervullina and $E$. maxima are produced named as Paracox 5 and Paracox 8 (UK) and Supercox (China), it is a mixed strain vaccine consisting of precocious strain of E. tenella and non-attenuated strains of E. acervulina and E. maxima ${ }^{(2,17)}$. The commercial success of this type of vaccine lies primarily in breeder and layer flocks where anti-coccidial drugs have been banned to prevent the carryover of drugs into eggs and meat ${ }^{(15)}$.

b) Vaccines based on drug-abbreviated infections. This vaccination technique was developed in 1970 and involves an infection and treatment strategy. Hemoprotozoan parasite infections are not self-limiting, and parasites can proliferate continuously in the blood stages if not checked by the immune response or drug treatment. Solid, sterile immunity develops after primary infection, and vaccination of cattle by infection with pathogenic wild-type Theileria parva followed by drug treatment (long-acting tetracyclines) has been used for many years to control East Coast fever ${ }^{(15)}$. In India, live sporozoite vaccine of Theileria annulata from infected ticks accompanied by chemo immunoprophylaxis with tetracycline or buparvaquone is formulated ${ }^{(18)}$. Protection is thought to be conferred mainly by cellmediated immunity, more specifically, CD8cytotoxic T-lymphocytes, against the intracellular schizont ${ }^{(19)}$.

c) Vaccines based on infections with parasites with a truncated life cycle cysts are produced by several protozoans within the host that are a persistent source of infection when eaten by other animals. These cysts can also cause reinfection when the immune system is compromised or can be reactivated during pregnancy, causing congenital disease, and abortion. Toxoplasma gondii infects a wide variety of hosts, including humans and is the major cause of abortion in sheep and goats. As immunity to primary infection develops, the intracellularly replicating tachyzoites become encysted in a dormant stage (zoitocysts), which can persist for several years, containing hundreds of infective bradyzoites. $T$. gondii parasites that were continuously passage in mice to produce diagnostic antigens were later found to have lost their ability to form cysts. The S-48 strain of $T$. gondii is used as a live vaccine with a trade name of Toxovax and is currently only commercial protozoan vaccine for toxoplasmosis. S-48 is a laboratory adapted strain that has lost the ability to differentiate into bradyzoites (20). The "incomplete" S48 strain of $T$. gondii forms the basis of a commercial vaccine conferring long-lasting immunity (approximately 18 months) of susceptible ewes against Toxoplasma-induced abortion when administered prior to mating (21). As S-48 strain undergoes limited multiplication within host animal and thus stimulates an appropriate cell-mediated immune response involving CD4+, CD8+ $\mathrm{T}$ cells, and IFN, therefore not permitting the parasite to persist. This vaccine primes the immune system so as to prevent the spread of parasite within lymphatic systems and thus limiting the spread to the circulation of host. The vaccine is normally administered prior to mating and there is a meat and milk withdrawal period of 6 weeks following vaccination $^{(18)}$.

d) Vaccines based on virulence-attenuated strains Attenuated cell line vaccines against various blood protozoans like Babesia bovis, Babesia bigemina, T. annulata, Anaplasma spp. has been used successfully in a number 
of countries, e.g. Isarel, Iran, Morocco, Tunisia, India, China, etc. As reviewed by $(22,23)$, attenuated, but still immunogenic piroplasms of $B$. bovis and $B$. bigemina obtained after continuous passaging in splenectomized calves, live vaccines using infected blood collected from acute infections of splenectomized calves were developed in Australia several decades ago and are still used in most countries to protect against babesiosis. This vaccine sometimes supplemented with Anaplasma centrale infected blood where Anaplasma marginale is enzootic. Attenuated cell line vaccine of $T$. annulata are used in India and protection is engendered by the attenuated schizont vaccine which has been evaluated by laboratory challenge with live infected ticks or with ground up tick sporozoites inoculated through syringe passage. The results of these challenges vary from no clinical response at all to mild transient clinical reaction with parasitemia. This attenuated schizont vaccine was commercialized under the trade name of "RakshaVac T" and is produced and marketed by Indian Immunological at Hyderabad, India (18). A continuous exposure to natural tick infections is generally required to ensure continuous and long-lasting immunity through these virulence attenuated vaccines. To increase shelf life and safety frozen-blood vaccines stored in liquid nitrogen using either dimethyl sulfoxide or glycerol as the cryoprotectant are produced ${ }^{(15)}$. Killed and Subunit Protozoan Vaccines Several inactivated vaccines consisting of crude whole organisms or, more recently, defined antigenic structures are available and are registered for companion animal market. In general, these vaccines are not as effective as live organisms, but can mitigate disease or transmission to various degrees. They may form the basis for the development of recombinant vaccines. The challenge in developing effective killed vaccine is to select an appropriate parasitic antigen and delivers it to the immune system in such a form that it should have the capacity to induce effective innate and adaptive immune response ${ }^{(18)}$.

\section{Killed vaccines}

Neospora caninum is a major cause of abortion in cattle (intermediate host). A crude $N$. caninum vaccine consisting of inactivated $N$. caninum tachyzoites with an adjuvant available in the United States to aid in the reduction of $N$. caninum induced abortion in healthy pregnant cattle and prevents the transmission of the parasite to calves in-utero. Neogaurd is the trade name of this vaccine ${ }^{(24,25)}$. Giardia intestinalis is an enteric parasite of many animal species and can cause severe GI disease in young and immune compromised individuals. Giardia Vax® a commercial vaccine has been licensed for use in dogs and cats in USA and it is commercialized to significantly reduce the incidence, severity, and duration of cyst shedding. The vaccine consists of a crude preparation of disrupted, axenically cultured G. intestinalis trophozoites (sheep isolate) ${ }^{(26,}$ 27). Marsh (28), evaluated and proposed a vaccine that alleviates equine protozoal myeloencephalitis caused by Sarcocystis neurona. Epm Vaccine consists of in-vitro cultured merozoites, which are chemically inactivated, originally obtained from the spinal cord of a horse, and mixed with an appropriate adjuvant. Trichguard vaccine is a killed vaccine containing axenic culture in broth containing killed trophozoites in adjuvant against Tritrichomonas foetus for cattle $^{(29)}$.

\section{Subunit and recombinant vaccines}

Subunit and recombinant vaccine consist of certain key molecules that manipulate the host immune response for their survival ${ }^{(30)}$ and thus by negating the function of these molecules prevent the establishment of 
parasite in the host. Substantial approaches have been achieved in recent years for the development of subunit and recombinant vaccines ${ }^{(31-33)}$. Theileria sporozoite and schizont antigens have been identified for development of subunit vaccines. Sporozoite antigens are surface antigens (SAGs) of both Theileria parva (p67) and $T$. annulata. Recombinant immunodominant sporozoite gene for T. annulata, i.e., SPAG-1 antigen has also been identified and tested, but all these antigens do not provide protection in a required level ${ }^{(18)}$. Two subunit vaccines against canine babesiosis have been developed. Both vaccines consist of soluble parasite antigens (SPA) that are released into the culture supernatant by in-vitro -cultured parasites, combined with adjuvant. The first vaccine is, Pirodog®, contains SPA from Brucella canis cultures only ${ }^{(34)}$, whereas recently developed is NobivacPiro ${ }^{\circledR}$ which contains SPA from B. canis and Babesia rossi in an attempt to broaden the strain-specific immunity. The protective effect of this vaccine seems to be based on the antibodydependent neutralization of a soluble parasite substance ${ }^{(35)}$. A killed vaccine approach was also formulated for $T$. gondii by using different recombinant proteins like membrane-associated SAGs, dense granules antigens rhoptry proteins, and micronemal proteins ${ }^{(14)}$.

Subunit vaccine against E. maxima is commercialized in Israel and it consists of two purified gametocyte antigens, i.e., GAM56 and GAM 82. This vaccine results in the induction of maternal immunity which is boosted by natural infection. Immunized laying hens transfer maternal antibody $\operatorname{IgY}$ to chicks ${ }^{(17,36)}$.

"Leishmune" a subunit vaccine has been registered in Brazil against visceral leishmaniosis. It consists of a glycoprotein complex isolated from Leishmania donovani, fucose mannose ligand, and a saponin adjuvant. This vaccine is also useful in blocking transmission and thus is able to control zoonotic visceral leishmaniosis ${ }^{(18)}$. CaniLeish provisionally licensed in parts of Europe and contain excreted and secreted proteins of Leishmania infantum ${ }^{(37)}$. Lemesre et al., (38), showed $92 \%$ efficacy of this vaccine over a period of 2 years. Leish-Tecis licensed in Brazil since 2007 and is only recombinant vaccine for protozoa containing Leishmania amastigote antigen (A2) purified from transfected Escherichia coli.

\section{Helminth vaccines}

There are three different families of helminthes nematodes (roundworms), trematodes (flatworms), and cestodes (tapeworms). These are multicellular parasites having genetic complexity, and there physical size, cannot be internalized by phagocytic cells of the immune system or killed by classical cytotoxic $\mathrm{T}$ cells. Hence, immune system has to develop a new mechanism referred to as the Type 2 or allergic-type immune response, typified by the recruitment and activation of potent effector leukocytes, mast cells, and eosinophils ${ }^{(39,40)}$.

\section{Vaccines against cestodes}

Vaccines developed against cestodes are recombinant vaccines, which are based on antigens of the parasite stage that adhere to the gut wall and induce immune responses that interfere with successful attachment. In 1990s, effective recombinant vaccines were developed against the cestodes Taenia ovis, Taenia saginata, Taenia solium, and Echinococcus granulosus ${ }^{(12)}$. To date, only the vaccine against the cestode $T$. ovis has been registered in Australia and New Zealand, but has not been marketed. The antigens are $45 \mathrm{~W}$, TSOL-18, TSOL-45 and EG-95 for $T$. ovis, T. solium, E. granulosus, respectively all 
these are Oncosphere proteins and interferes with attachment ${ }^{(12,44)}$.

\section{Vaccines against trematodes}

The most important disease in terms of geographical distribution and economic losses to farmers is Fasciolosis caused by Fasciola hepatica and F. gigantica ${ }^{(41)}$. According to Raina ${ }^{(41)}$, no viable vaccine are present because of the variable efficacy between animal species and the protection level to date is $<80 \%$. Limited number of candidate antigen is known for Fasciola spp. and no viable vaccine has been developed with protection level more than $80 \%$. Various vaccine candidates have been identified as key targets for vaccination against Fasciolosis. These are Cathepsin-B and Cathepsin-L, which are present in excretory and secretory materials of Fasciola spp., these are then expressed in yeast and used as a multivalent vaccine in a rat model. Vaccination of Friesian cattle with recombinant $F$. hepatica cathepsin L-1 protease formulated in Montanide $70 \mathrm{VG}$ and 206 VG was done and the animals were exposed to fluke contaminated pasture for 13 weeks and there was a reduction of $48.2 \%$ in fluke burden as compared to control and nonvaccinated groups. Other vaccine candidates are leucine amino-peptidase (LAP) present in bacteria, plants, and animals and are novel drug targets and vaccine candidates in parasitic infections ${ }^{(42)}$.

Partial purification and characterization of LAP from $F$. hepatica and vaccination in sheep has shown $89 \%$ protection. Piacenza ${ }^{(42)}$ formulated a cocktail of LAP, CL-1, and CL2 which gave $78 \%$ protection. However, production of viable vaccine is difficult as the parasite has the ability to target similar host pathways and has evolved its own strategy to confound host defenses ${ }^{(43)}$.

\section{Vaccines against nematodes}

There are two strategies for vaccine development against GI nematodes attenuated vaccines and hidden antigen $\operatorname{approach}^{(45)}$.

\section{Attenuated vaccines}

The first and most effective vaccine against lungworms was a $\times 1000-$ an irradiated larval vaccine against Dictyocaulus viviparous infection in cattle named "DICTOL" and is commercially available and marketed in Europe. The vaccine was developed ${ }^{(46)}$ and contains irradiated $\mathrm{L}_{3}$-larvae that do not mature to adult worms. It is administered in two doses of attenuated larvae and induced up to $98 \%$ protection against challenge infection. In India, vaccine against sheep lungworm Dictyocaulus filaria was developed using a similar approach of $50 \mathrm{k} \mathrm{R}$ gamma irradiated attenuated $\mathrm{L}_{3}$ larvae and was named "DIFIL" ${ }^{(47)}$. A similar approach was used to develop a vaccine against the canine intestinal nematode Ancylostoma caninum ${ }^{(48)}$, but it was observed that irradiation-attenuated larval vaccines developed against GI nematodes did not protect young, susceptible stock against infection and were, therefore, never commercialized $^{(49)}$. Moreover, larvae must be harvested from the manure of infected animals, so these vaccines are difficult to produce too.

\section{Vaccines based on hidden antigens}

Hidden antigens are integral membrane proteins associated with the gut of GI nematodes. Hidden antigens discovered against $H$. contortus are $\mathrm{H} 11, \mathrm{H}$-gal-P, cysteine proteases, and Enolase (45). The principle of immunization is that recombinant hidden antigens molecules induce a high level of antibody which cross-reacted with native antigen molecules. 


\section{Ecto-parasite vaccines}

Ectoparasitic arthropods would seem to be the ultimate challenge in vaccine development, as they not only are large and complex but also spend most of their life outside or on the surface of the host. Of all the ectoparasites ticks poses a serious threat in livestock industries. They are obligate hematophagous arthropods that parasitize every vertebrate and are responsible for transmission of various tick-borne diseases and can also cause severe toxic conditions, paralysis, and allergy (50). Vaccine against the cattle tick, Rhiphicepahalus (Boophilus) microplus, is a recombinant vaccine based on a protein (Bm86) found in the tick at the surface of the gut wall. It was first introduced commercially in Australia in 1994 (TickGUARD) ${ }^{(51)}$. This vaccine is unique in that it is not based on natural antigens recognized by the immune system during infection, but takes advantage of the ferocious blood-feeding habits of the tick. Vaccination stimulates the production of high antibody levels in cattle against a tick gut membrane-bound protein, $\mathrm{Bm} 86$, using a recombinant protein in a potent adjuvant. These antibodies bind to the tick's gut surface when taking a blood meal, causing the rupture of the gut wall, and tick death ${ }^{(52)}$. The vaccine induces significant levels of protection against tick infestation and, in some cases, against tick-borne diseases. However, antibody levels are not boosted by infection and need to be maintained at high levels by repeated immunization. Use of vaccine in conjunction with drug administration limits its practical and commercial use. Moreover, the presence of a tick immunoglobulin excretion system hampers the effectiveness of this vaccine approach in other ticks ${ }^{(53)}$. Another vaccine for tick Gavac which is a Cuban vaccine contain recombinant Rhiphicepahalus (Boophilus) miroplus Bm86 gut antigen expressed in Pichia pastoris. This is 99\% effective against Rhiphicepahalus (Boophilus) miroplus and is $100 \%$ effective against Rhiphicepahalus (Boophilus) annulatus ${ }^{(53)}$.

\section{Edible vaccines}

The edible vaccines are a novel approach for sustainable development of vaccines for prophylaxis. According to $(54,55)$ vaccine, genes are expressed in edible plants, which are consumed by animals. As a major alternative, plants are emerging as a promising system to express and manufacture a wide range of functionally active parasitic antigens (56). Plants like alfalfa, tobacco, lettuce, potato, maize rice, banana, wheat, corn carrots, peanuts, and soybeans have been studied for vaccine production. The major advantages of edible vaccines are that once plants are created there seeds can be used for stored for future use, these are cheaper to manufacture and there is no need of purification and processing as the final product are edible (2). With so many advantages the drawbacks of edible vaccines are there genetic variation, batch to batch variation unexpression of vaccine candidates in plants, poor immunogenicity of certain vaccines in the host, not suitable for all types of animals, and there can be development of oral tolerance to the antigens ${ }^{(2)}$.

In conclusion, Future of developing vaccines for control of parasitic infections in animals is bright and is most sustainable and economical approach. There is need of maintaining synergism between parasitic vaccines and anti-parasitic drugs as this will help in the reduction of dependence on anti-parasitic drugs, thus decreasing the development of resistance. Traditional methods of control of parasites like chemotherapy, grazing management, biological control of parasites can be used in an integrated pattern for efficient control but are not feasible economically in many countries including India. Vaccines offer long-lived immunity that can be repeatedly boosted when animals 
are exposed to natural infections. The first most effective vaccine against a parasitic disease was an irradiated larval vaccine against $D$. viviparous infection in cattle named DICTOL and on the same principle of irradiation first parasitic vaccine in India was against Dictyocaulus filaria (Lungworm of sheep) named DIFIL (1981). In the field of helminth vaccines, various subunit vaccines have been developed using various candidate antigens but due to their evasion mechanism from the host immunity development, vaccines for helminthes is still in progress. Various live vaccines are produced against protozoan parasites and are licensed and commercialized to large scale in many countries including India, but these vaccines poses a risk of reversion of non-virulent or attenuated strain to virulent strain in immunecompromised animal leading to failure of vaccination and live vaccine also have short shelf life and need cold chain to deliver. In spite of these drawbacks live schizont vaccine of $T$. annulata is a successful commercialized vaccine in India with a brand name of RakshaVac-T formulated by Indian Immunologicals, Hyderabad. Vaccines against zoonotic and foodborne parasites $(T$. ovis, T. solium, Echinococcus multilocularis, poultry Coccidia etc.) are important as they are aimed at reducing risk for the consumer and are also responsible for increase in production. A number of vaccines have been formulated and commercialized in various parts of the world regarding this aspect like Coccivac B, Coccivac D, Immunocox C1, Paracox, etc. vaccine against $T$. ovis containing $45 \mathrm{~W}$ subunit antigens. There are many hurdles in vaccine development due to immune evasion capacity of parasites, antigenic diversity but advances in field of molecular biology, proteomics, immunology, and recombinant technologies have provided a way for the development of vaccines for animal parasites.

\section{References}

1. Balic A, Bowles VM and Meeusen EN. "The immunobiology of gastrointestinal nematode infections in ruminants." 2000:181-241.

2. Baltzell P, Newton $\mathrm{H}$ and O'Connor AM. A Critical Review and Meta- Analysis of the Efficacy of Whole- Cell Killed Tritrichomonas foetus Vaccines in Beef Cattle. Journal of veterinary internal medicine 2013; 27(4):760-70.

3. Bandyopadhyay S, Mandal S, Datta KK, Devi P, De S, Bera AK et al., Economic analysis of risk of gastrointestinal parasitic infection in cattle in North Eastern States of India. Tropical animal health and production 2010; 42(7):1481-6.

4. Buxton D. Toxoplasmosis: the first commercial vaccine. Parasitology today 1993; 9(9):335-7.

5. Chen J, Huang SY, Zhou DH, Li ZY, Petersen E, Song HQ et al., DNA immunization with eukaryotic initiation factor- $2 \alpha$ of Toxoplasma gondii induces protective immunity against acute and chronic toxoplasmosis in mice. Vaccine 2013; 31(52):6225-31.

6. Dalgliesh RJ, Callow LL, Mellors LT and McGregor W. Development of a highly infective Babesia bigemina vaccine of reduced virulence. Australian Veterinary Journal 1981; 57(1): 8-11.

7. Dalton JP, Robinson MW, Mulcahy G, O'Neill SM and Donnelly S. Immunomodulatory molecules of Fasciola hepatica: candidates for both vaccine and immunotherapeutic development. Veterinary parasitology 2013; 195(34):272-85.

8. De Waal DT and Combrink MP. Live vaccines against bovine babesiosis. Veterinary parasitology. 2006; 138(12):88-96.

9. Dhar DN and Sharma RL. Immunization with irradiated larvae against Dictyocaulus filaria in young lambs. Veterinary Parasitology 1981; 9(2):125-31. 
10. EMA. European Medicines Agency, Scientific discussion of Cani Leish 2011.

11. Garcia-Garcia JC, Montero C, Redondo M, Vargas M, Canales M, Boue $\mathrm{O}$ et al., Control of ticks resistant to immunization with Bm86 in cattle vaccinated with the recombinant antigen $\mathrm{Bm} 95$ isolated from the cattle tick, Boophilus microplus. Vaccine 2000; 18(21):2275-87.

12. Garg R, Kundu K, Kumar S and Banerjee PS. Current trends and future prospects of vaccine development against poultry coccidiosis. Molecular Biological Approaches for Diagnosis and Control of Parasitic Diseases. Indian Veterinary Research Institute, Izatnagar, U.P., India. 2013; 182.

13. Hayashida KY, Abe TA, Weir WI, Nakao RY, Ito KI, Kajino KI et al., Wholegenome sequencing of Theileria parva strains provides insight into parasite migration and diversification in the African continent. DNA research 2013; 20(3):20920.

14. Hewitson JP, Grainger JR and Maizels RM. Helminth immunoregulation: the role of parasite secreted proteins in modulating host immunity. Molecular and Biochemical Parasitology. 2009; 167(1):1-1.

15. Holroyd N and Sanchez- Flores A. Producing parasitic helminth reference and draft genomes at the Wellcome Trust Sanger Institute. Parasite immunology 2012; 34(2- 3):100-7.

16. Ibelli AM, Ribeiro AR, Giglioti R, Regitano LC, Alencar MM et al., Resistance of cattle of various genetic groups to the tick Rhipicephalus microplus and the relationship with coat traits. Veterinary Parasitology 2012; 186(34):425-30.

17. Innes EA, Bartley PM, Rocchi $M$, Benavidas-Silvan J, Burrells A, Hotchkiss E et al., Developing vaccines to control protozoan parasites in ruminants: dead or alive?. Veterinary Parasitology 2011; 180(1-2):155-63.

18. Innes EA, Wright S, Bartley P, Maley S, Macaldowie C, Esteban-Redondo I et al.,
The host-parasite relationship in bovine neosporosis. Veterinary immunology and immunopathology 2005; 108(1-2):29-36.

19. Jacob SS, Cherian S, Sumithra TG, Raina OK and Sankar M. Edible vaccines against veterinary parasitic diseases-current status and future prospects. Vaccine 2013; 31(15):1879-85.

20. Jarrett WF, Jennings FW, McIntyre WI, Mulligan $\mathrm{W}$ and Urquhart GM. Immunological studies on Dictyocaulus viviparus infection. Immunity produced by the administration of irradiated larvae. Immunology 1960; 3(2):145-51.

21. Kalyanasundaram R. Role of parasite vaccines in sustained animal health and production. Journal of Veterinary Parasitology 2015; 29(2):73-83.

22. Karim SA, Porwal K, Kumar S and Singh VK. Carcass traits of Kheri lambs maintained on different system of feeding management. Meat Science 2007; $76(3): 395-401$.

23. Kloosterman A, Parmentier $\mathrm{HK}$ and Ploeger HW. Breeding cattle and sheep for resistance to gastrointestinal nematodes. Parasitology Today 1992; 8(10):330-5.

24. Knox DP and Smith WD. Vaccination against gastrointestinal nematode parasites of ruminants using gut-expressed antigens. Veterinary Parasitology. 2001; 100(12):21-32.

25. Knox DP. Development of vaccines against gastrointestinal nematodes. Parasitology 2000; 120:43-61.

26. Kumar B, Nagar G, Shakya M and Ghosh $\mathrm{S}$. Cross protective vaccine development against ticks. Molecular Biological Approaches for Diagnosis and Control of Parasitic Diseases. Indian Veterinary Research Institute, Izatnagar, U.P., India 2013; 82 .

27. Kumar CS, Deepesh G, Mahavir Y and Archana T. Edible vaccine: a new platform for the development of malaria vaccine. Critical Reviews ${ }^{\mathrm{TM}}$ in Eukaryotic Gene Expression 2012; 22(3).

28. Kumar T, Tufani NA, Prasad A, Arora N and Rajora VS. Veterinary parasitic 
vaccines-A current scenario. International Journal of Livestock Reserch 2013; 3(1):511.

29. Larsen M. Biological control of helminths. International Journal for Parasitology 1999; 29(1):139-46.

30. Lemesre JL, Holzmuller P, Gonçalves RB, Bourdoiseau G, Hugnet C, Cavaleyra M et $a l$., Long-lasting protection against canine visceral leishmaniasis using the LiESApMDP vaccine in endemic areas of France: double-blind randomised efficacy field trial. Vaccine 2007; 25(21):4223-34.

31. Lightowlers MW and Heath DD. Immunity and vaccine control of Echinococcus granulosus infection in animal intermediate hosts. Parassitologia 2004; 46(1-2):27-31.

32. Maizels RM, Balic A, Gomez-Escobar N, Nair $M$ and Taylor MD. Helminth parasites: masters of regulation. Immunological Review 2004; 751(201):89116.

33. Marsh AE, Lakritz J, Johnson PJ, Miller MA, Chiang Y and Chu H. Evaluation of immune responses in horses immunized using a killed Sarcocystis neurona vaccine. Veterinary Therapeutics 2004; 5(1):34-42.

34. McKeever DJ, Taracha EL, Morrison WI, Musoke AJ and Morzaria SP. Protective immune mechanisms against Theileria parva: evolution of vaccine development strategies. Parasitology Today. 1999;15(7):263-7.

35. Meeusen EN, Walker J, Peters A, Pastoret $P P$ and Jungersen G. Current status of veterinary vaccines. Clinical microbiology reviews 2007;20(3):489-510.

36. Menon R, Gasser RB, Mitreva $M$ and Ranganathan S. An analysis of the transcriptome of Teladorsagia circumcincta: its biological and biotechnological implications. InBMC genomics 2012, 13(7): 10.

37. Miller TA. Industrial development and field use of the canine hookworm vaccine. In Advances in Parasitology 1978; 16: 333342.

38. Moreau Y, Vidor E, Bissuel $G$ and Dubreuil N. Vaccination against canine babesiosis: an overview of field observations. Transactions of the Royal Society of Tropical Medicine and Hygiene 1989; 83:95-6.

39. Nisbet AJ, McNeilly TN, Wildblood LA, Morrison AA, Bartley DJ, Bartley Y et al., Successful immunization against a parasitic nematode by vaccination with recombinant proteins. Vaccine 2013; 31(37): 4017-23.

40. Nuttall PA, Trimnell AR, Kazimirova M and Labuda M. Exposed and concealed antigens as vaccine targets for controlling ticks and tick- borne diseases. Parasite immunology 2006; 28(4):155-63.

41. Olson ME, Ceri H and Morck DW. Giardia vaccination. Parasitology today 2000; 16(5):213-7.

42. Piacenza L, Acosta D, Basmadjian I, Dalton JP and Carmona C. Vaccination with cathepsin $\mathrm{L}$ proteinases and with leucine aminopeptidase induces high levels of protection against fascioliasis in sheep. Infection and immunity 1999; 67(4):195461.

43. Prasad A and Sankar M. Immunoprophylaxis against GI nematodes in small ruminants. Molecular Biological Approaches for Diagnosis and Control of Parasitic Diseases. Indian Veterinary Research Institute, Izatnagar, U.P., India 2013;182.

44. Raina OK, Samanta S, Jacob S, Yadav HS, Chandra S and Bouri RK. Current status of vaccine development against Fasciolosis. Molecular Biological Approaches for Diagnosis and Control of Parasitic Diseases. Indian Veterinary Research Institute, Izatnagar, U.P., India 2013; 182.

45. Romero JJ, Perez E and Frankena K. Effect of a killed whole Neospora caninum tachyzoite vaccine on the crude abortion rate of Costa Rican dairy cows under field conditions. Veterinary parasitology 2004; 123(3-4):149-59.

46. Sanyal PK, Chauhan JB and Mukhopadhyaya PN. Implications of fungicidal effects of benzimidazole compounds on Duddingtonia flagrans in integrated nematode parasite management 
in livestock. Veterinary research communications 2004; 28(5):375-85.

47. Saravanan BC, Ray DD and Sankar M. Conventional and molecular vaccine against protozoans infecting livestock. Molecular Biological Approaches for Diagnosis and Control of Parasitic Diseases. Indian Veterinary Research Institute, Izatnagar, U.P., India. 2013; 182.

48. Schetters T. Vaccination against canine babesiosis. Trends in Parasitology 2005; 21(4):179-84.

49. Shalaby HA. Anthelmintics resistance; how to overcome it?. Iranian Journal of Parasitology 2013; 8(1):18.

50. Singh D and Swarnkar CP. Epidemiology and pasture management for the control of ovine gastrointestinal nematodosis. InProceedings of FAO Symposium on "Integrated Animal Parasite Management: From Academic Interest to Reality" 2005:6-8.

51. Singh D, Swarnkar CP, Khan FA, Srivastava CP and Bhagwan PS. Epidemiology of ovine gastrointestinal nematodes at an organised farm in Rajasthan, India. Small Ruminant Research
1997; 26(1-2):31-7.

52. Sutherland IA and Leathwick DM. Anthelmintic resistance in nematode parasites of cattle: a global issue?. Trends in parasitology $2011 ; 27(4): 176-81$.

53. Vercruysse J, Knox DP, Schetters TP and Willadsen P. Veterinary parasitic vaccines: pitfalls and future directions. Trends in Parasitology. 2004;20(10):488-92.

54. Wallach M, Smith NC, Petracca M, Miller $\mathrm{CM}$, Eckert $\mathrm{J}$ and Braun R. Eimeria maxima gametocyte antigens: potential use in a subunit maternal vaccine against coccidiosis in chickens. Vaccine 1995; 13(4):347-54.

55. Waller PJ. From discovery to development: current industry perspectives for the development of novel methods of helminth control in livestock. Veterinary parasitology 2006; 139(1-3):1-4.

56. Willadsen P, Bird P, Cobon GS and Hungerford J. Commercialization of a recombinant vaccine against Boophilus microplus. Parasitology 1995; 110: 43-50.

\section{How to cite this article:}

Hardeep Kalkal, Sukhdeep Vohra and Snehil Gupta. 2020. Importance of Parasitic Vaccines in Integrated Control of Parasitic Diseases in Livestock. Int.J.Curr.Microbiol.App.Sci. 9(02): 2036-2048. doi: https://doi.org/10.20546/ijcmas.2020.902.232 